\title{
Exploring Phylogeny at the Tree of Life Web Project
}

\author{
Adam M. Goldstein
}

Published online: 16 October 2010

(C) Springer Science+Business Media, LLC 2010

\begin{abstract}
The University of Arizona's Tree of Life Web Project organizes information about the biological taxa on the model of the tree of life itself. This creates intuitive and informative pathways for browsing. The Project has a well-articulated protocol for checking on the quality of information published on the site, which is evaluated a number of times as it is added to the site. This paper is intended to be an entree to the site for teachers at all levels and the general public.
\end{abstract}

Keywords Phylogeny • Tree of life •

Information architecture •

Organization of information - Teaching •

Internet resources $\cdot$ Biological taxa

\section{Introduction}

The only image in Darwin's Origin (1859) depicts a phylogenetic tree: lineages split and diverge; the divergent lineages do the same; repeating the pattern, the hierarchy of biological taxa is created. The tree of life image as a representation of evolution finds its full expression on the Internet. Computer users can traverse the tree of life in its entirety, from its base at the domain or kingdom level to its most recent leaves at the species level. Moreover, the tree creates its own framework for organizing information about itself. Branches on the tree image represent points at which daughter and

\footnotetext{
A. M. Goldstein $(\varangle)$

Department of Philosophy, Iona College,

715 North Avenue, New Rochelle,

NY 10801, USA

e-mail: z_californianus@shiftingbalance.org
}

parent lineages diverge. Information about the origin of a major new group is best presented on the diagram at the branching points or at tips, which represent a major group's terminus, whether it persists into the present or is extinct. The University of Arizona's Tree of Life Web Project (http://tolweb.org) is distinguished by its elegant, useful, and authoritative digital publication of information about phylogeny using the tree of life image. The central aim of this paper is to offer educators and researchers an entrée to the site. After a brief account of the project's history, background, and authority, I describe elements of its architecture and information content likely to be of interest to educators, and the site's tools for creating content and sharing it with others. I conclude by pointing to some other worthwhile web resources for learning about phylogeny.

\section{History and Background}

On its "about" page, ${ }^{1}$ The Tree of Life Web Project (ToL), is described as follows.

The Tree of Life Web Project is a collection of information about biodiversity compiled collaboratively by hundreds of expert and amateur contributors. Its goal is to contain a page with pictures, text, and other information for every species and for each group of organisms, living or extinct. Connections between Tree of Life web pages follow phylogenetic branching patterns between

\footnotetext{
${ }^{1} \mathrm{http} / /$ tolweb.org/tree/home.pages/abouttol.html; facts mentioned in the paragraph below are all from this source, unless otherwise noted.
} 
groups of organisms, so visitors can browse the hierarchy of life and learn about phylogeny and evolution as well as the characteristics of individual groups.

The Project began as a personal project of David Maddison, a faculty member in the University of Arizona's Department of Entomology and Department of Ecology and Evolutionary Biology. Maddison, in the course of his Ph.D. research in the late 1980s, wrote a computer program for phylogenetic analysis, MacClade, still available at http://macclade.org. MacClade's purpose was to provide a way for computer users to browse phylogenetic trees on their computers by hyperlinking information about higher and lower taxa. A decade or so later, during which work on MacClade had slowed, the program was updated to organize files on the World Wide Web. The aim was to integrate database records distributed around the world into a phylogeny browser that could be used to explore, in a seamless manner, all of the taxa. This goal was realized in 1995; a year later, approximately 20 pages had been added, authored by people other than the Project's founders, and stored on machines located outside of the University of Arizona campus. The site was formally launched in January 1996. In July 2000, the National Science Foundation funded the design of a new database architecture and its implementation, the first major funding for the project. Web pages for taxa at a range of hierarchical levels have been added at a steady rate since 1996, contributed by more than 320 biologists from 21 countries. The Project now has a full-time staff of five, including David Maddison, a managing editor and technical designer, two programmers, and a server administrator. Users can visit the "Tree of Life Growth Monitor" (http://tolweb.org/tree/home.pages/growth.html) to see what content has been added since 1996-"more than 10,000 WWW pages," according to the Project home page.

\section{Architecture and Information Content}

The information on ToL, as I mention above, is organized on the same plan as the tree of life itself. From the site's home page, users can begin exploring the tree from several points that users will find natural and intuitive. Starting at the base of the tree, users are given the option of following branches deriving from taxa at the kingdom level (see Fig. 1). As is generally the case on the site, where there are significant disagree- ments in the scientific community about substantive matters, alternative representations of the branching point are presented and explained; in this case, there is an issue about the number and nature of taxa at the kingdom level. Other points of entry into the tree of life allow the user to focus on a particular taxon: by searching, a taxon of the user's own choosing; by following links to popular taxa, recently added pages, or for the adventurous, a randomly selected taxon. Once they find a starting point in the tree of life from which to begin browsing, users can begin following the pathways created by evolution itself.

The primary bearers of information are the web pages for each taxon. There are pages for major junctions, such as phyla. From these pages, a user can follow lineages passing through the junction, moving to the species level. These pages display an image of the organisms in the daughter taxa showing the segment of the tree of life at which the branch occurs. Some pages are placeholders, which provide links and references to scientific literature. These are marked "temporary page" in the upper-right-hand corner. Pages whose content has been added have the character of a detailed encyclopedia entry about the taxon. Authors address topics germane to the page's taxon; for instance, the Acanthostega gunnari page has sections on the taxon's phylogenetic position, skull, sensory organs, postcranial skeleton, and paleoecology and lifestyle (see Figs. 2 and 3). A page for an extant taxa would, of course, have more information about other topics, such as ecology, behavior, geographical distribution, life history, and soft part anatomy.

\section{The Project's Authority}

ToL has two central mechanisms for ensuring the quality of the information it publishes. Firstly, authors are highly qualified. Most are faculty members in science departments, and hold a Ph.D. Museum curators, who have training and experience commensurate with university faculty, are also well-represented. A page's author and his or her institutional affiliation are listed at the footer of each page, and in some cases, there are also links to their personal web pages, which can also be found at http://tolweb.org/onlinecontributors/ app? service $=$ page $/$ ScientificContributorsPage .

Secondly, ToL pages are subject to a process of peer review. Placeholder pages, mentioned above, are created by ToL editors, and are not reviewed. Once an author begins work on a new page, it is labeled "under construction." It is not reviewed, and is 


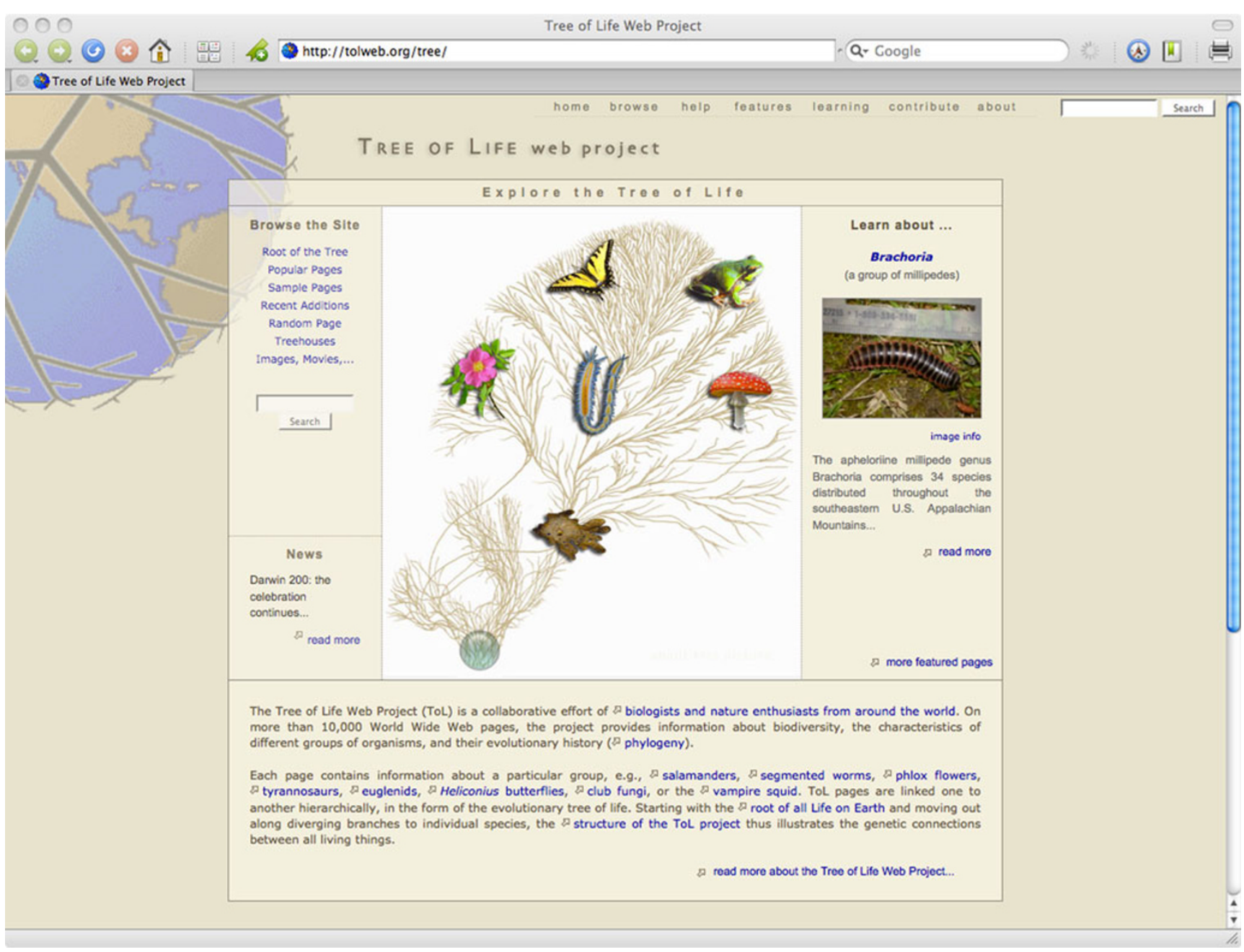

Fig. 1 The ToL home page, at http://tolweb.org. Accessed 4 October 2010

considered provisional. When the author and ToL editors agree that the page has reached a stable point covering the important aspects of the taxon, it is marked "complete." Because an editor's approval is required before a page is considered complete, complete pages do undergo a preliminary form of review. These pages do not usually cover the taxon in great depth, consisting of a sparse list of the identifying traits of organisms in the taxon and a brief discussion of the taxon's place in the tree of life. So the preliminary review provides some assurance that the page can be trusted, even if that assurance is not definitive. There are two further standards a ToL page can meet: "tol-reviewed" and "peer-reviewed." Tol-reviewed pages are approved by a ToL coordinator. A ToL coordinator is responsible for pages about a subject area, about which he or she is a specialist. These reviews are not blinded; both author and reviewer are known to one another. Pages meeting the standard for peer review are evaluated by at least two scientists selected for their expertise in the subject area of the contribution, and who are not ToL staff members, and who are not known to the author. The details of these processes are explained on the site's section for quality control (http:// tolweb.org/tree/home.pages/quality.html).

Because information about the taxa is created by experts and, for those pages that reach the tol-reviewed stage, evaluated by subject-area experts, a site user can be confident that ToL scientific content accurately reflects the current state of opinion about the subjects treated. Pages reaching the peer-review stage are evaluated in a way that promotes the reviewer's ability to frankly assess the page content, because the reviewer remains anonymous. The ToL review process is 


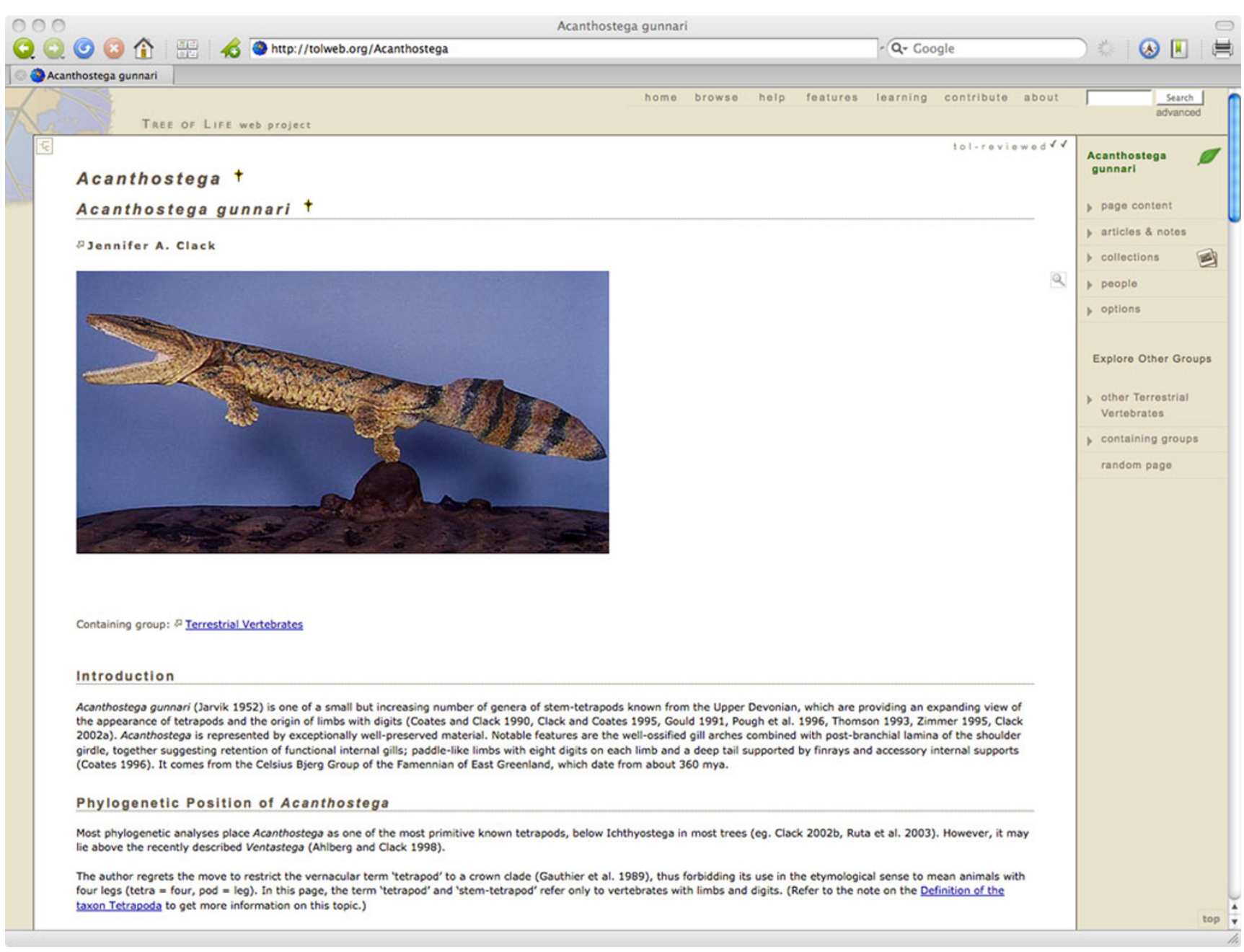

Fig. 2 ToL page for Acanthostega gunnari, indicating the general characteristics of a page at the site for a taxon. Page: http://www.tolweb.org/Acanthostega. Accessed 4 October 2010.
Note the annotation in the upper right, "tol-reviewed." See "The Project's Authority" for more information about this and other annotations concerning quality control explained in detail, and the status of a page is clearly indicated, so there is no ambiguity about how much scrutiny the page has been subject to. ToL outperforms almost all scientific journals, which generally do not publish the steps in the review process, and which in general do not indicate, on each article, how much scrutiny it has undergone. The safeguards intrinsic in ToL's protocols give the user the ability to assess the credibility of a ToL page, and to feel confident that pages passing the later stages of review are accurate.

\section{Creating and Sharing}

ToL is a community space, in which users of all levels can share web pages of their own invention. In a space separated off from the pages for taxa created by ToL science professionals, users can add pages to sections of the site organized by the purpose and structure of their content (Visit http://tolweb.org/ tree/home.pages/treehouses.html; also, see Fig. 4). In the section with room for a wide range of web page topics and aims, users can create a page with the same general format as the pages used to display information about taxa on the ToL site. Using an editing environment much like those used to create Wiki pages, users can create their own section headings, adding content in the resulting section. Users can also bring together pages about different taxa from the static taxon pages on the main site. This allows users to create a page with a theme. For example, there are treehouses about clownfish, dogs, and retroviruses, created by middle and high school students, college students, graduate students in the sciences, and professional scientists. 


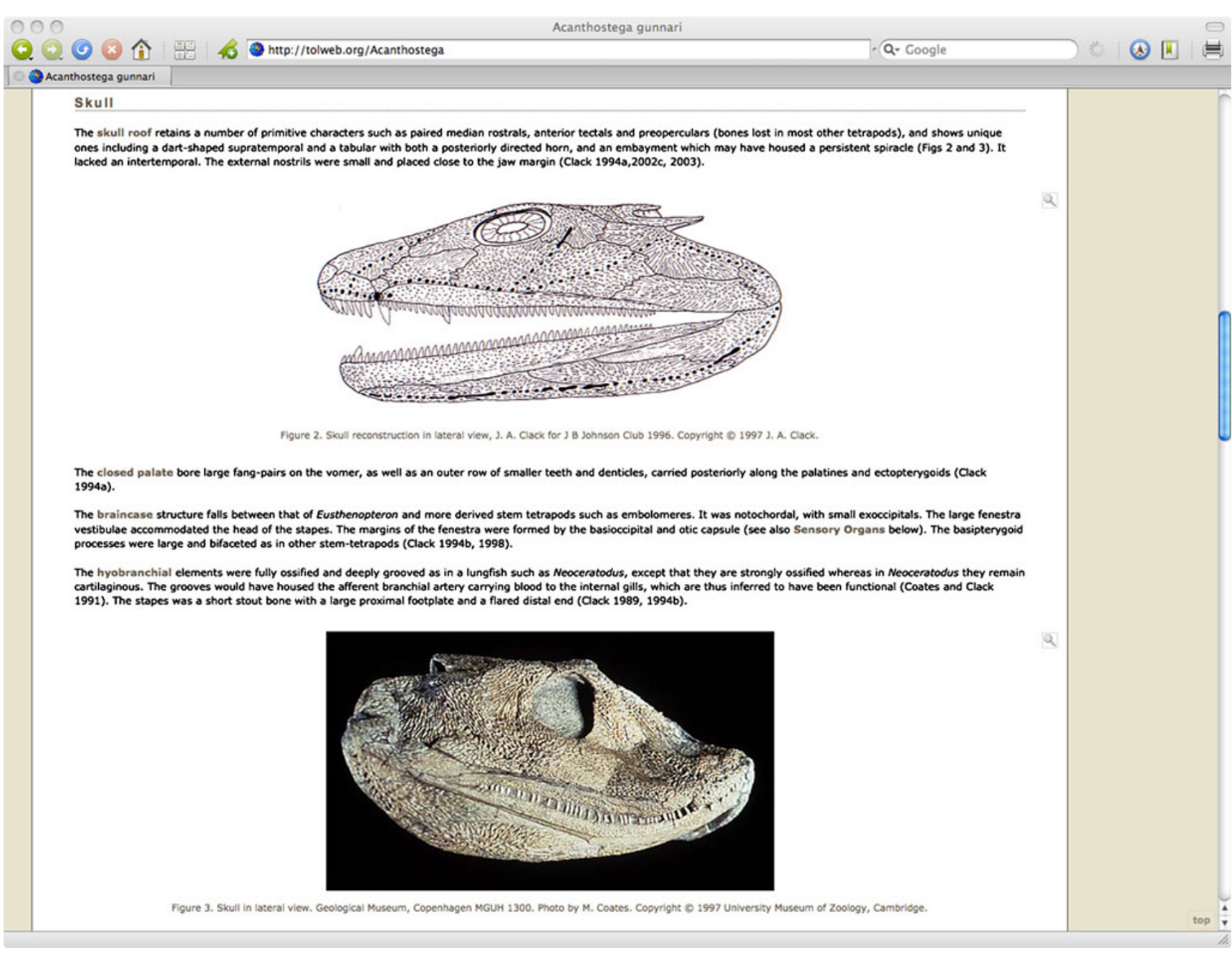

Fig. 3 The Acanthostega gunnari page, showing the organization of a ToL taxon page into sections, which differ from page to page, depending on the nature of the taxon. Illustrations and color photographs appear frequently. Page: http://www.tolweb. org/Acanthostega. Accessed 4 October 2010
The other sections in the user-created part of the site include Fun and Games, Stories, Art and Culture, and Portfolios. These sections are aimed at younger audiences, although the portfolio section would be useful to people of all ages. In the Fun and Games section, someone has created a set of flashcards for studying about the taxa. "Miss Blue Morpho's Story Time," in the Stories section, is a story about the butterfly life cycle, suitable for elementary school students. It is illustrated in color. The Art and Culture section contains a guide to animal origami, instructions for building a mobile, and words and music for songs such as "The Sea Star Song." Portfolios collects the work of a group, for instance, a group of students in a class who have each created their own treehouses. A Teacher Resources section contains links to lesson plans incorporating treehouse building; there is also a lengthy section with treehouse building activities focused on Arizona.

Users must register to create treehouses; name and email address are required. This is necessary for creating an account on the site for saving work and maintaining treehouses. Anyone over 18 years old can register; those under 18 years old must register as a part of a classroom group.

\section{Other Online Resources of Note}

The idea of creating a web site with a web page for every taxon is not new, and ToL is not the only such site being developed. The University of Michigan's Animal Diversity Web site, at http://animaldiversity. ummz.umich.edu/site/index.html, only covers animals, 


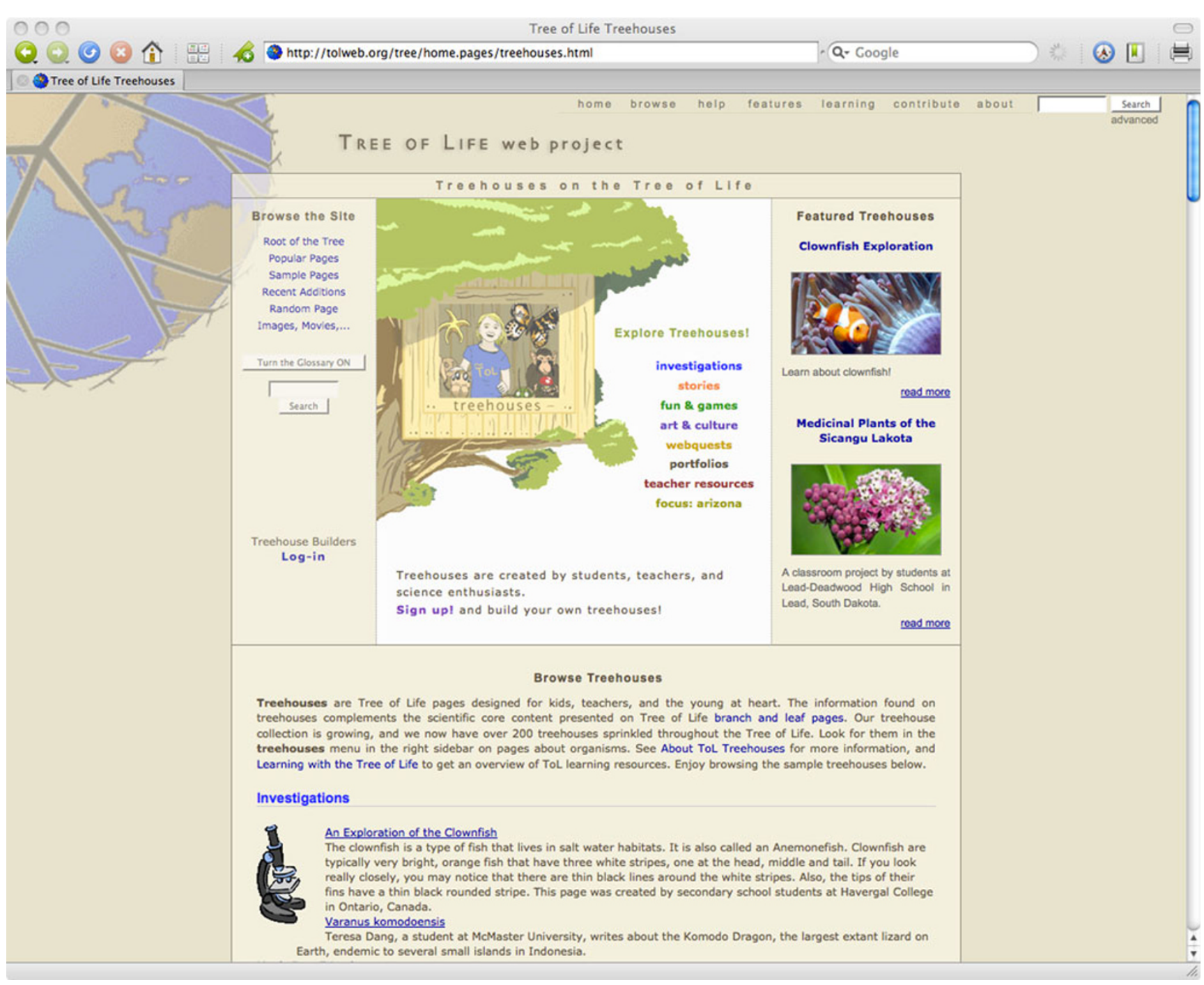

Fig. 4 Portal to ToL's sharing and community-building section, at http://tolweb.org/tree/home.pages/treehouses.html. Accessed 4 October 2010

although the breadth of its coverage of them is extensive. There are resources for college and $\mathrm{K}-12$ instructors. A rich page of links to other sites about animal species, conservation, and diversity is especially useful (http://animaldiversity.ummz.umich.edu/site/help/ relatedLinks.html). The copyright notice is dated from 1995, although no information is available on the site about how long it has been in operation. The latest entry in the News section, dated November 2009, is about the implementation of a new search technology at the site, suggesting that development is continuing.

The Encyclopedia of Life (EOL; http://www.eol.org) is a new project which aims to have a single web page for each species. It has the support of well-known scientists, E. O. Wilson being among its chief spokespeople. Every page on the EOL site is rich with images, links, menus, and logos, with the dynamic content and design one would expect of a corporate web site. The tree of life is represented in a small window in the form of a list, lower-level taxa names indented below higher-level taxa. This is not as effective at representing evolutionary relationships among the taxa, but is rather most useful for representing taxonomy as a way of categorizing groups within groups, a practice far older than Darwin. Each EOL page, each of which represents a single taxon, has links to information on other sites, for instance, linking to the NCBI databases, Wikipedia, and to other sites with information about taxonomic names. The integration of the EOL site with these other data sources is an impressive technological accomplishment.

Each page is created initially by a professional scientist, although, much like Wikipedia, there are tools for users in the general public to add content to each 
species page. A professional scientist, a curator, is given responsibility for a page or set of pages. Curators are expected to monitor EOL content as it is added; information about the curator for a particular page can be found by following a link in the page's footer. Material created with teachers in mind can be found at http://education.eol.org/educators/ed_start; at present, this page is lacking in significant content, describing the kind of resources that are expected to become available as the site grows. As with the ToL treehouse, the EOL provides an editing environment for grouping pages about the taxa to create a custom section of the EOL site about a theme of a user's invention.

\section{Concluding Remarks}

The hierarchical organization of biological taxa is understood at present, in a Darwinian framework, to result from relationships of descent. Taxa categorized together, for instance, species in the same phylum, have diverged less from a shared common ancestor than taxa categorized in different higher-level groups. These relationships of descent suggest a theme for organizing information about the taxa: branching points represent the point of origin for diverging biological individuals which do not share the same evolutionary fate; these branching points also represent, in the visual image of a reticulate tree, an ideal point at which to place information about these new individuals. The Tree of Life Web Project has used just this model as the basis for the architecture of its web site. Accordingly, that site is an excellent resource for students, teachers, and anyone else interested in phylogeny.

\section{Reference}

Darwin C. On the origin of species by means of natural selection, or the preservation of favoured races in the struggle for life (London: John Murray). In: Mayr E, editor. On the origin of species: a facsimile of the first edition, 1964, reprinted. Massachusetts: Harvard University Press; 1859. 